\title{
Proof of conjecture in: The basic reproduction number obtained from Jacobian and next generation matrices - A case study of dengue transmission modelling
}

\author{
Hyun Mo Yang ${ }^{1 *}$ and David Greenhalgh ${ }^{2}$ \\ ${ }^{1}$ UNICAMP - IMECC - DMA \\ Praça Sérgio Buarque de Holanda, 651 \\ CEP: 13083-859, Campinas, SP, Brazil, \\ ${ }^{2}$ Department of Mathematics and Statistics, University of Strathclyde, \\ Livingstone Tower, 26 Richmond Street, \\ Glasgow, G1 1XH, UK.
}

\begin{abstract}
The spectral radius of the next generation matrix provides an expression for the basic reproduction number. Instead of calculating the dominant eigenvalue of the characteristic equation corresponding to the next generation matrix, a threshold parameter can be obtained by handling the coefficients of this equation. Here we prove two conjectures presented in [9].
\end{abstract}

Keywords: Compartmental modelling - Routh-Hurwitz criteria - Spectral radius Threshold - Single infection - Coinfection

\section{Introduction}

In [9], from the Jacobian and next generation matrices, the stability of the disease free equilibrium (DFE) was assessed. Briefly, the application of the Routh-Hurwitz criteria to the coefficients of the characteristic equation corresponding to the Jacobian matrix $F$ evaluated at the DFE resulted in the determination of a possible basic reproduction number $R_{0}^{\dagger}$. But, the spectral radius corresponding to the next generation matrix $F_{1} V^{-1}$ resulted in, for instance, $\rho\left(F_{1} V^{-1}\right)=\sqrt{R_{0}^{\dagger}}$, where $R_{0}^{\dagger}$ is that obtained from the Jacobian method and $F=F_{1}-V$, with $F_{1}$ being the transmission matrix and $V$, the transition matrix [1], [2]. In both methods, if $R_{0}^{\dagger}<1$ or $\rho\left(F_{1} V^{-1}\right)<1$, the DFE is locally asymptotically stable (LAS).

${ }^{*}$ Corresponding author: hyunyang@ime.unicamp.br; tel/fax: + 5519 3521-6031 
Instead of calculating the spectral radius of the next generation matrix $\rho\left(F_{1} V^{-1}\right)$, a threshold parameter can be obtained from the characteristic equation corresponding to the matrix $F_{1} V^{-1}$. The absolute sum of the negative coefficients is the threshold. With respect to this approach, in [9] two conjectures were presented without proofs. Here, we present the proofs.

\section{Proofs of two conjectures}

Conjecture 1: Let the characteristic polynomial of order $n$ corresponding to the next generation matrix $F_{1} V^{-1}$ be written as

$$
\Lambda(\lambda)=\lambda^{n}-a_{n-1} \lambda^{n-1}-\cdots-a_{1} \lambda-a_{0}
$$

with $a_{i} \geq 0$, for $i=0,1,2, \ldots, n-1$. Let $R_{0}$ denote the spectral radius of the next generation matrix, that is, $R_{0}=\rho\left(F_{1} V^{-1}\right)$, and

$$
R_{0}^{*}=a_{n-1}+\cdots+a_{1}+a_{0}
$$

Then $R_{0}^{*}$ is a threshold value for the disease to take off or die out in the sense that:

(i) $\quad R_{0}^{*}>1$ if and only if $R_{0}>1$,

(ii) $\quad R_{0}^{*}=1$ if and only if $R_{0}=1$,

and

(iii) $\quad R_{0}^{*}<1$ if and only if $R_{0}<1$.

Proof: If all $a_{i}=0$, the result is obvious. Otherwise the elements of the next generation matrix $F_{1} V^{-1}$ are non-negative as they correspond to expected numbers of different types of infected individuals. Hence, by the Perron Frobenius Theorem [8] it has a non-negative right eigenvector whose eigenvalue is $R_{0}$, and $R_{0}$ is the largest real eigenvalue. Additionally, the characteristic polynomial (1) is such that the number of sign differences between consecutive nonzero coefficients is one. Hence, according to Descartes rule of signs, there is exactly one positive root. However, writing

$$
\Lambda(\infty)=\lim _{\lambda \rightarrow \infty} \Lambda(\lambda)
$$

(i) if $R_{0}^{*}>1$, we have $\Lambda(1)<0$ and $\Lambda(\infty)=\infty$, so $\Lambda(\lambda)$ has a root in $(1, \infty)$. Hence, the unique largest real eigenvalue $R_{0}$ corresponding to the characteristic polynomial (1) is $R_{0}>1$.

(ii) if $R_{0}^{*}=1$, we have $\Lambda(0)<0, \Lambda(1)=0$ and $\Lambda(\infty)=\infty$, so the unique positive root of $\Lambda(\lambda)=0$ is $\lambda=1$, and $R_{0}=1$.

(iii) if $R_{0}^{*}<1$, we have $\Lambda(0)<0$ and $\Lambda(1)>0$, so $\Lambda(\lambda)$ has a root in $(0,1)$. Hence, the unique largest real eigenvalue $R_{0}$ corresponding to the characteristic polynomial (1) is $R_{0}<1$.

Therefore $R_{0}^{*}$ is a valid threshold parameter that crosses the value one exactly when $R_{0}$ does and determines the disease behaviour in the same way that $R_{0}$ does. The application of the Routh-Hurwitz criteria to the characteristic equation corresponding to the Jacobian matrix 
$F=F_{1}-V[9]$, showed that the DFE is LAS if $R_{0}^{\dagger}<1$, (equivalently $R_{0}<1$, see Section 3 of this paper), and unstable if $R_{0}^{\dagger}>1$, equivalently $R_{0}>1$.

The above conjecture was obtained considering a single infection. Another conjecture deals with coinfection.

Conjecture 2: When two infections occur, let the characteristic polynomial corresponding to the next generation matrix $F_{1} V^{-1}$ be written as

$$
\Lambda(\lambda)=\Lambda_{1}(\lambda) \Lambda_{2}(\lambda)-\Lambda_{3}(\lambda)
$$

where $\Lambda_{1}(\lambda)$ and $\Lambda_{2}(\lambda)$, given by equation (1), are the characteristic polynomials of degree $n$ corresponding to single infection by infections 1 and 2 respectively, and $\Lambda_{3}(\lambda)$ is the characteristic polynomial of degree $n-1$ involving coinfection given by

$$
\Lambda_{3}(\lambda)=a_{n-1}^{3} \lambda^{n-1}+\cdots+a_{1}^{3} \lambda+a_{0}^{3},
$$

with $a_{i}^{3} \geq 0$, for $i=0,1,2, \ldots, n-1$.

(a) If $\Lambda_{3}(\lambda) \equiv 0$, then $R_{0}^{*}=\max \left\{R_{0}^{1 *}, R_{0}^{2 *}\right\}$ is a threshold value for disease to take off or die out in the sense that conditions (3)-(5) hold.

(b) If $\Lambda_{3}(\lambda) \not \equiv 0$, then define $R_{3}^{*}=\Lambda_{3}(1)=a_{n-1}^{3}+\cdots+a_{1}^{3}+a_{0}^{3}$, analogous to $R_{0}^{*}$ defined by (2), but for coinfection, and

$$
R_{t}^{*}=\frac{R_{3}^{*}}{\left(1-R_{0}^{1 *}\right)\left(1-R_{0}^{2 *}\right)},
$$

(possibly infinite). Then

$$
R_{0}^{*}=\max \left\{R_{0}^{1 *}, R_{0}^{2 *}, R_{t}^{*}\right\}
$$

(also possibly infinite) is a threshold value for disease to take off or die out in the sense that conditions (3)-(5) hold.

Proof: (a) First, when $\Lambda_{3}(\lambda) \equiv 0$, Conjecture 1 can be applied for each infection. As $R_{0}$ is the largest real positive eigenvalue of the characteristic equation,

$$
R_{0}=\max \left\{R_{0}^{1}, R_{0}^{2}\right\}
$$

when the result follows from Conjecture 1.

(b) When $\Lambda_{3}(\lambda) \not \equiv 0$, the product $\Lambda_{1}(\lambda) \Lambda_{2}(\lambda)$, one term of the characteristic polynomial (6), is such that $\Lambda_{1}(0) \Lambda_{2}(0)=a_{0}^{1} a_{0}^{2} \geq 0$. The other term $\Lambda_{3}(\lambda)$ is a strictly increasing function (or a constant, if $\Lambda_{3}(\lambda)=a_{0}^{3}$ ), with $\Lambda_{3}(0)=a_{0}^{3}$. Recall that $\Lambda_{i}(\lambda)$ has a unique positive root $R_{0}^{i}$ for $i=1,2$. Hence, one of the roots, $r_{s}$ (which can be negative if $a_{0}^{1} a_{0}^{2}<a_{0}^{3}$ ), of $\Lambda(\lambda)=0$ is less than or equal to the minimum of the roots $R_{0}^{1}$ and $R_{0}^{2}$ of $\Lambda_{1}(\lambda)=0$ and $\Lambda_{2}(\lambda)=0$ respectively and another $\left(r_{b}\right)$ is strictly greater than $\max \left\{R_{0}^{1}, R_{0}^{2}\right\}$. Again as $F_{1} V^{-1}$ is a next generation matrix its elements are non-negative and $R_{0}$ is its largest real eigenvalue. Hence $R_{0} \geq r_{b}$ is strictly greater than $\max \left\{R_{0}^{1}, R_{0}^{2}\right\}$. By Lemma 1 (below), $R_{0}=r_{b}$, so

$$
R_{0}>\max \left\{R_{0}^{1}, R_{0}^{2}\right\} .
$$


If $R_{0}^{*}>1$ then either $(i) \max \left\{R_{0}^{1 *}, R_{0}^{2 *}\right\}>1,($ ii $) \max \left\{R_{0}^{1 *}, R_{0}^{2 *}\right\}=1$ and $R_{t}^{*}>1$, or (iii) $\max \left\{R_{0}^{1 *}, R_{0}^{2 *}\right\}<1$ and $R_{t}^{*}>1$. In the first case, by Conjecture $1 \max \left\{R_{0}^{1}, R_{0}^{2}\right\}>1$ so $R_{0}>1$ by (9). In the second case similarly, $\max \left\{R_{0}^{1}, R_{0}^{2}\right\}=1$, so $R_{0}>1$ by (9). In the third case

$$
\begin{aligned}
\Lambda(1) & =\Lambda_{1}(1) \Lambda_{2}(1)\left[1-\frac{\Lambda_{3}(1)}{\Lambda_{1}(1) \Lambda_{2}(1)}\right] \\
& =\left(1-R_{0}^{1 *}\right)\left(1-R_{0}^{2 *}\right)\left(1-R_{t}^{*}\right),
\end{aligned}
$$

where $R_{t}^{*}$ is given by equation (7), and $\Lambda(\infty)=\infty$ due to the assumption that the degree of the polynomial $\Lambda_{3}(\lambda)$ is less than the other two. As $R_{t}^{*}>1$ we have $\Lambda(1)<0$ and $R_{0}$ lies in $(1, \infty)$ so $R_{0}>1$.

If $R_{0}^{*}=1$ and $\max \left\{R_{0}^{1 *}, R_{0}^{2 *}\right\}=1$ then $R_{t}^{*}=\infty$ which contradicts $R_{0}^{*}=1$. Hence $\max \left\{R_{0}^{1 *}, R_{0}^{2 *}\right\}<1$ and $R_{t}^{*}=1$ so arguing as in the third case above $\Lambda(1)=0$. Moreover by Conjecture $1, \max \left\{R_{0}^{1 *}, R_{0}^{2 *}\right\}<1$ implies that $\max \left\{R_{0}^{1}, R_{0}^{2}\right\}<1$ so by using Lemma 1 , $R_{0}=1$. Hence we have shown that $R_{0}^{*}=1$ implies that $R_{0}=1$.

If $R_{0}^{*}<1$ then $\max \left\{R_{0}^{1 *}, R_{0}^{2 *}\right\}<1$ and $R_{t}^{*}<1$, hence $\Lambda(1)>0$. Again by Conjecture 1 , $\max \left\{R_{0}^{1}, R_{0}^{2}\right\}<1$ and by Lemma 1 the unique root of $\Lambda(\lambda)$ in $\left(\max \left\{R_{0}^{1}, R_{0}^{2}\right\}, \infty\right)$ is less than one. So we have shown that $R_{0}^{*}<1$ implies that $R_{0}<1$.

So once again $R_{0}^{*}$ is a valid threshold parameter that crosses the value one exactly when $R_{0}$ does and determines the disease behaviour in the same way that $R_{0}$ does. The condition $\max \left\{R_{0}^{1 *}, R_{0}^{2 *}, R_{t}^{*}\right\}<1$ can be written as

$$
\max \left\{R_{0}^{1 *}, R_{0}^{2 *}\right\}<1-R_{3}^{*} \quad \text { and } \quad R_{t}^{*}<1
$$

(see Lemma 2).

Lemma $1 \Lambda(\lambda)$ has exactly one positive real root in $\left(\max \left\{R_{0}^{1}, R_{0}^{2}\right\}, \infty\right)$.

Proof Note that at $R_{0}^{i}, i=1,2, \Lambda\left(R_{0}^{i}\right)=-\Lambda_{3}\left(R_{0}^{i}\right)<0$. Hence $\Lambda\left(\max \left\{R_{0}^{1}, R_{0}^{2}\right\}\right)<0$ and $\Lambda(\infty)=\infty$ so there is a positive real root of $\Lambda(\lambda)$ in $\left(\max \left\{R_{0}^{1}, R_{0}^{2}\right\}, \infty\right)$. Note also that

$$
\Lambda_{i}(\lambda)=\lambda^{n}\left(1-\frac{a_{n-1}^{i}}{\lambda}-\frac{a_{n-2}^{i}}{\lambda^{2}}-\cdots-\frac{a_{0}^{i}}{\lambda^{n}}\right),
$$

and that for $\lambda \geq R_{0}^{i}$ both $\lambda^{n}$ and

$$
\left(1-\frac{a_{n-1}^{i}}{\lambda}-\frac{a_{n-2}^{i}}{\lambda^{2}}-\cdots-\frac{a_{0}^{i}}{\lambda^{n}}\right)
$$

are positive monotone increasing functions, so $\Lambda_{i}(\lambda)$ is positive monotone increasing. Hence $\Lambda_{1}(\lambda) \Lambda_{2}(\lambda)$ is also positive monotone increasing in $\left(\max \left\{R_{0}^{1}, R_{0}^{2}\right\}, \infty\right)$. But

$$
\begin{aligned}
\Lambda(\lambda) & =\Lambda_{1}(\lambda) \Lambda_{2}(\lambda)\left[1-\frac{\Lambda_{3}(\lambda)}{\Lambda_{1}(\lambda) \Lambda_{2}(\lambda)}\right] \\
& =\Lambda_{1}(\lambda) \Lambda_{2}(\lambda)\left[1-\frac{\Lambda_{3}(\lambda) / \lambda^{2 n}}{\left\{\Lambda_{1}(\lambda) / \lambda^{n}\right\}\left\{\Lambda_{2}(\lambda) / \lambda^{n}\right\}}\right]
\end{aligned}
$$


In $\left(\max \left\{R_{0}^{1}, R_{0}^{2}\right\}, \infty\right), \Lambda_{1}(\lambda) / \lambda^{n}$ and $\Lambda_{2}(\lambda) / \lambda^{n}$ are both monotone increasing and strictly positive and $\Lambda_{3}(\lambda) / \lambda^{2 n}$ is strictly monotone decreasing and strictly positive, hence

$$
1-\frac{\Lambda_{3}(\lambda) / \lambda^{2 n}}{\left\{\Lambda_{1}(\lambda) / \lambda^{n}\right\}\left\{\Lambda_{2}(\lambda) / \lambda^{n}\right\}}
$$

is strictly monotone increasing. So $\Lambda(\lambda)$ is strictly monotone increasing in $\left(\max \left\{R_{0}^{1}, R_{0}^{2}\right\}, \infty\right)$. The result follows.

Lemma 2 The condition $\max \left\{R_{0}^{1 *}, R_{0}^{2 *}, R_{t}^{*}\right\}<1$ is equivalent to $\max \left\{R_{0}^{1 *}, R_{0}^{2 *}\right\}<1-R_{3}^{*}$ and $R_{t}^{*}<1$.

Proof The statement is obvious if $R_{3}^{*}=0$ so suppose that $R_{3}^{*}>0$. When $\max \left\{R_{0}^{1 *}, R_{0}^{2 *}\right\}<1$, $R_{t}^{*}$, given by equation (7), is a strictly increasing function in $R_{0}^{1 *}$ and $R_{0}^{2 *}$, with value $R_{3}^{*}$ when $R_{0}^{1 *}=0$ and $R_{0}^{2 *}=0$. Hence, $R_{t}^{*}<1$ implies that $R_{3}^{*}<1$.

Consider $R_{0}^{2 *}=1-R_{3}^{*}+\varepsilon$, with $|\varepsilon| \approx 0$. Then

$$
R_{t}^{*}=\frac{R_{3}^{*}}{\left(1-R_{0}^{1 *}\right)\left[1-\left(1-R_{3}^{*}+\varepsilon\right)\right]}=\frac{R_{3}^{*}}{\left(1-R_{0}^{1 *}\right)\left(R_{3}^{*}-\varepsilon\right)} .
$$

When $R_{0}^{1 *}=0$, we have

$$
R_{t}^{*}=\frac{R_{3}^{*}}{R_{3}^{*}-\varepsilon}\left\{\begin{array}{lll}
>1, & \text { if } & \varepsilon>0 \\
<1, & \text { if } & \varepsilon<0
\end{array}\right.
$$

We deduce that in general (provided that $\max \left\{R_{0}^{1 *}, R_{0}^{2 *}\right\}<1$ ) if $R_{0}^{2 *} \geq 1-R_{3}^{*}$, then $R_{t}^{*} \geq 1$, and $\max \left\{R_{0}^{1 *}, R_{0}^{2 *}, R_{t}^{*}\right\}<1$ is not satisfied. Similarly if $R_{0}^{1 *} \geq 1-R_{3}^{*}$, then $\max \left\{R_{0}^{1 *}, R_{0}^{2 *}, R_{t}^{*}\right\} \geq 1$. Hence, $\max \left\{R_{0}^{1 *}, R_{0}^{2 *}, R_{t}^{*}\right\}<1$ implies that $\max \left\{R_{0}^{1 *}, R_{0}^{2 *}\right\}<1-R_{3}^{*}$, with $R_{3}^{*}<1$ and $R_{t}^{*}<1$. On the other hand if $\max \left\{R_{0}^{1 *}, R_{0}^{2 *}\right\}<1-R_{3}^{*}$ and $R_{t}^{*}<1$, then clearly $\max \left\{R_{0}^{1 *}, R_{0}^{2 *}, R_{t}^{*}\right\}<1$.

\section{Conclusion}

In this brief paper we have proved two conjectures. For the second conjecture the case $\Lambda_{3}(\lambda) \equiv 0$ may describe infections where infection with each disease or strain individually does not influence the infection with the other disease or strain. Another possibility is when each infection confers complete immunity against the other. In this case $\left(\Lambda_{3}(\lambda) \equiv 0\right)$ both infections die out when $R_{0}^{*}=\max \left\{R_{0}^{1 *}, R_{0}^{2 *}\right\}<1$. On the other hand, the condition for co-existence of two non-interacting infections is given by $\min \left\{R_{0}^{1 *}, R_{0}^{2 *}\right\}>1$.

However for the case $\Lambda_{3}(\lambda) \not \equiv 0$, we would expect that coinfection can occur, even when $\max \left\{R_{0}^{1 *}, R_{0}^{2 *}\right\}<1$, provided that $R_{t}^{*}>1$. In other words, a sufficiently high interaction between strains sustains both infections and the DFE is unstable. For this reason, besides $\max \left\{R_{0}^{1 *}, R_{0}^{2 *}\right\}<1$ we must have $R_{t}^{*}<1$, or $R_{0}^{*}=\max \left\{R_{0}^{1 *}, R_{0}^{2 *}, R_{t}^{*}\right\}<1$, in order for the DFE be LAS. An example of coinfection where the DFE is LAS when the largest real eigenvalue $R_{0}$ of $F_{1} V^{-1}$ is strictly less than one is found in [6]. Indeed Raimundo et al. [6], dealing with coinfection with drug sensitive and drug resistant tuberculosis, showed that the DFE is LAS if $\max \left\{R_{0}^{1}, R_{0}^{2}\right\}<1-R_{3}^{*}$ and $R_{t}^{*}<1$, with $R_{3}^{*}<1$. 
In spectral radius theory, the dominant eigenvalue of the characteristic equation of the next generation matrix $F_{1} V^{-1}$, which is the spectral radius $\rho\left(F_{1} V^{-1}\right)$, determines the stability of the DFE in the sense that:

(i) $\rho\left(F_{1} V^{-1}\right)<1$ if and only if all eigenvalues of the matrix $F=F_{1}-V$ have strictly negative real part,

(ii) $\rho\left(F_{1} V^{-1}\right)=1$ if and only if zero is an eigenvalue of $F$,

and

(iii) $\rho\left(F_{1} V^{-1}\right)>1$ if and only if $F$ has a strictly positive real eigenvalue.

In the proofs of our two conjectures, using the same characteristic equation corresponding to the next generation matrix $F_{1} V^{-1}$, we developed a new threshold $R_{0}^{*}$, given by equations (2) and (8), which was determined to be a threshold equivalent to $R_{0}$ in that the DFE was LAS if $R_{0}^{*}<1$ and unstable if $R_{0}^{*}>1$. These new definitions are not spectral radii, and we therefore cannot directly establish the stability or instability of the DFE from that property. However in [9], the stability of the DFE was assessed by the Routh-Hurwitz criteria applied to the Jacobian matrix $F$ (if all Routh-Hurwitz criteria are satisfied, then all eigenvalues have strictly negative real parts). But the arguments above show that the values $R_{0}^{*}$ given in (2) and (8) are equivalent to $R_{0}^{\dagger}$ as thresholds determining the stability of the system and thus equivalent to the Jacobian method in assessing stability. Hence for both (2) and (8) here the disease is LAS if $R_{0}^{*}<1$ and unstable if $R_{0}^{*}>1$. Thus the stability threshold from the Jacobian method is also the same as the results given by (2) and (8).

We are grateful to a referee for pointing out that this method has potential applications in other disease models, in particular brucellosis in sheep and cattle.

Sun and Zhang [7] discuss a sheep brucellosis model with immigration. From p.339 the corresponding characteristic equation is

$$
\lambda^{3}-A_{11} \lambda^{2}=0
$$

where $R_{0}^{*}=R_{0}=A_{11}=\frac{S_{0}(d+m+\alpha)}{(d+m)(d+\alpha)}\left(\beta+\frac{\beta_{1} k}{n \tau+\epsilon}\right)$, from equation (2). Nie et al. [5] discuss a different model for cattle brucellosis in Jinlin province, China, and there our method can be applied in a similar fashion.

Li et al. [3] discuss a model for the spread of brucellosis between sheep and cattle in a public farm. From the matrix $F V^{-1}$ on p.586 of [3] the corresponding characteristic equation is

$$
\Lambda(\lambda)=\left(\lambda-A_{11}\right)\left(\lambda-A_{22}\right) \lambda^{4}-A_{12} A_{21} \lambda^{4}=0,
$$

where $A_{11}, A_{22}, A_{12}$ and $A_{21}$ are constants as defined by [3], with $R_{0}^{1}=A_{11}$ and $R_{0}^{2}=A_{22}$.

For the models with one strain only

$$
\text { and } \begin{aligned}
\Lambda_{1}(\lambda) & =\left(\lambda-A_{11}\right) \lambda^{2}, \\
\Lambda_{2}(\lambda) & =\left(\lambda-A_{22}\right) \lambda^{2},
\end{aligned}
$$

so the characteristic equations satisfy the hypotheses of Conjecture 2 with $\Lambda_{3}(\lambda)=A_{21} A_{12} \lambda^{4}$. Hence $R_{0}^{1}=R_{0}^{1 *}, R_{0}^{2}=R_{0}^{2 *}$ and 


$$
R_{t}^{*}=\frac{A_{12} A_{21}}{\left(1-R_{0}^{1}\right)\left(1-R_{0}^{2}\right)} .
$$

The stability condition is equivalent to $R_{0}^{*}=\max \left\{R_{0}^{1 *}, R_{0}^{2 *}, R_{t}^{*}\right\}<1$, or equivalently, $\max \left\{R_{0}^{1 *}, R_{0}^{2 *}\right\}<$ $1-A_{12} A_{21}$ and $R_{t}^{*}<1$.

Li et al. [4] discuss an alternate model for the spread of brucellosis amongst sheep and cattle in China. Here the characteristic equations do not quite satisfy the hypotheses of Conjecture 2 but a simple modification of the arguments in this paper show that the same method can be applied similarly as in [3].

\section{Acknowledgments}

Project supported through grant from FAPESP - Projeto temático, 2009/15098-0 (HMY) and CNpq grant 300981/2014-7 (DG).

\section{References}

[1] Diekmann, O., Heesterbeek, J.A.P., Roberts, M.G., 2010. The construction of nextgeneration matrices for compartmental epidemic models. J. R. Soc. Interface 7, 873-885.

[2] van den Driessche, P., Watmough, J., 2002. Reproduction numbers and sub-threshold endemic equilibria for compartmental models in disease transmission. Math. Biosci. 180, 29-48.

[3] Li, M.-T., Sun, G.-Q., Wu, Y.-F., Zhang, J., Jin, Z. (2014a) Transmission dynamics of a multi-group brucellosis model with mixed cross infection in public farm. Appl. Math. Comp. $237,582-594$.

[4] Li, M.-T., Sun, G.-Q., Zhang, J., Jin, Z., Sun, X.-D., Wang, Y.-M., Huang, B.-X, Zheng, Y.-H. (2014b) Transmission dynamics and control for a brucellosis model in Hinggan League of Inner Mongolia, China, Math. Biosci. Eng. 11(5), 1115-1137.

[5] Nie, J., Sun, G.-Q., Sun, X.-D., Zhang, J., Wang, N., Wang, Y.-M., Shen, C.-J., Huang, B.-X., Jin, Z. (2014) Modelling the transmission of dairy cattle brucellosis in Jilin Province, China, J. Biological Systems, 22(4), 533-554.

[6] Raimundo, S.M., Yang, H.M., Venturino, E., 2014. Theoretical assessment of the relative incidence of sensitive and resistant tuberculosis epidemic in presence of drug treatment. Math. Biosc. Eng. 11(4), 971-993.

[7] Sun, G.-Q., Zhang, Z.-K., 2014. Global stability for a sheep brucellosis model with immigration. Appl. Math. Comp. 246, 336-345.

[8] Varga, R.S., 2000, Matrix Iterative Analysis, Second Edition, Vol. 27, Springer Series in Computational Mathematics, Springer-Verlag, Berlin Heidelberg. 
[9] Yang, H.M., 2014. The basic reproduction number obtained from Jacobian and next generation matrices - A case study of dengue transmission modelling, Biosystems 126, 52-75. 